IdeAs

Idées d'Amériques

$6 \mid 2015$

Migrer dans les Amériques

\title{
Le retour de l'enfant prodigue. Les Québécois et l'élection fédérale canadienne de 2015
}

Louis Massicotte

\section{(2) OpenEdition}

\section{Journals}

Édition électronique

URL : https://journals.openedition.org/ideas/1257

DOI : 10.4000/ideas. 1257

ISSN : 1950-570

Éditeur

Institut des Amériques

Référence électronique

Louis Massicotte, «Le retour de l'enfant prodigue. Les Québécois et l'élection fédérale canadienne de 2015 », IdeAs [En ligne], 6 | 2015, mis en ligne le 17 décembre 2015, consulté le 20 octobre 2022. URL : http://journals.openedition.org/ideas/1257 ; DOI : https://doi.org/10.4000/ideas. 1257

Ce document a été généré automatiquement le 20 octobre 2022

\section{(c) (i) (9)}

Creative Commons - Attribution - Pas d'Utilisation Commerciale - Pas de Modification 4.0 International - CC BY-NC-ND 4.0

https://creativecommons.org/licenses/by-nc-nd/4.0/ 


\title{
Le retour de l'enfant prodigue. Les Québécois et l'élection fédérale canadienne de 2015
}

\author{
Louis Massicotte
}

\section{Introduction}

1 Lors des élections du 19 octobre 2015, les Libéraux ont défait de façon décisive les Conservateurs de Stephen Harper, au pouvoir depuis 2006. Défiant les prédictions, au terme d'une campagne d'une durée record de 78 jours, le parti de Justin Trudeau a remporté 184 sièges sur 338 , avec $39,5 \%$ des voix à l'échelle du pays, et fait élire des députés dans chacune des provinces. Pour la première fois depuis 1988, la majorité des sièges du Québec (40 sur 78) ${ }^{1}$ est allée au parti vainqueur dans l'ensemble du pays. L'aile fédérale du mouvement indépendantiste, le Bloc Québécois, a réalisé le pire score de son histoire, avec 19,3\% du vote. Tout s'est passé comme si les Québécois, après avoir boudé la gouverne du Canada depuis 1993, dans la foulée de l'échec de l'Accord du Lac Meech, avaient choisi de tourner la page et de réintégrer le giron politique canadien.

\section{Contexte}

2 Malgré ses indéniables succès au chapitre de l'économie et de la qualité de vie, le Canada a fait figure, au cours du dernier quart de siècle, de pays incertain (Brunet M. et Massicotte L.). L'hypothèse d'une sécession du Québec a longtemps pesé sur son avenir. Le sérieux de cette éventualité était attesté par le fait que de 1993 à 2011, la majorité des sièges fédéraux du Québec allait constamment au Bloc. Les Québécois francophones votaient sciemment pour un parti qui n'avait rigoureusement aucune chance d'exercer le pouvoir, ce qu'on pouvait lire comme une manifestation de dédain pour le régime fédéral lui-même. En se tournant en masse vers le Nouveau Parti Démocratique en 2011, 
les Québécois, tout en appuyant un parti fédéraliste, avaient encore une fois opté pour l'opposition.

3 Bien qu'ils aient été dirigés par des Québécois sous Jean Chrétien, Paul Martin et Stéphane Dion, de 1990 à 2008, les Libéraux avaient perdu leur emprise traditionnelle sur le Québec, sauf en 2000 où ils avaient fait élire 36 députés dans la province, contre 38 pour le Bloc québécois, surclassant même ce dernier au chapitre du vote populaire (44\% contre $40 \%$ ). Les ministres qui assuraient une présence forte du Québec dans le Cabinet libéral étaient pour la plupart élus dans des circonscriptions dominées par la minorité anglophone et les immigrants. L'affaire des commandites a fait voler en éclats ce fragile équilibre en discréditant les Libéraux. En 2006, les Conservateurs de Stephen Harper avaient réussi à obtenir au Québec le quart des voix et à faire élire 10 députés. Leur flirt avec le Québec, marqué notamment par l'adoption en 2006 d'une motion proclamant que les Québécois formaient une nation au sein d'un Canada uni, était fondé sur l'espoir d'une percée substantielle qui ne s'est pas matérialisée en 2008 (encore 10 sièges), et encore moins en 2011 (seulement 5). Le calibre des ministres québécois du gouvernement Harper n'était pas à la hauteur de celui de leurs prédécesseurs. Contrairement à l'ère Mulroney, personne ne s'est plaint sous Harper que le Québec ait dicté l'agenda politique du gouvernement.

4 Les efforts du Premier ministre Harper visant à conquérir le cœur des Québécois se sont heurtés à un mur à cause de l'incompatibilité radicale des valeurs conservatrices prédominant dans les provinces des Prairies et dans l'Ontario rural, avec la prédominance au Québec de la gauche et du centre-gauche. Rappelons que le Québec a l'un des taux de syndicalisation (40\%) parmi les plus élevés du continent, et une tradition bien ancrée d'interventionnisme gouvernemental. Le conservatisme moral et religieux qui caractérisait le Québec d'autrefois a fait place à un libéralisme moral plus prononcé qu'ailleurs en Amérique du nord. Contrairement aux progressistesconservateurs de l'ère Mulroney, le nouveau parti Conservateur était dirigé par un Albertain, incarnait un conservatisme plus radical évoquant celui des Républicains américains et surtout, préférait la rigueur idéologique au compromis. Les penchants monarchistes et militaristes du Premier ministre, devenus flagrants durant son dernier mandat, trouvaient peu d'échos au Québec. En compagnie des résidents des provinces de l'Atlantique, les Québécois ont été les Canadiens les plus réfractaires à son gouvernement, particulièrement durant la période où celui-ci a détenu la majorité des sièges (2011-2015).

5 Ce portrait doit bien sûr être nuancé, car le Québec n'est pas un bloc homogène. Comme ailleurs au Canada, les valeurs conservatrices y demeurent vivaces à l'extérieur des grands centres métropolitains, et les limites de l'étatisme paraissent plus évidentes à ceux qui n'en ont guère profité. Ce sentiment est particulièrement prononcé dans la ville de Québec et dans la région voisine de Chaudière-Appalaches, qui s'étend de la rive sud du Saint-Laurent à la frontière américaine.

6 La « vague orange » de 2011 avait pris de court tout le personnel politique et les médias. En pleine campagne, les aiguilles s'étaient soudainement mises à bouger pour le NPD. Lassitude à l'égard du Bloc québécois et du mouvement souverainiste? Réflexe de sympathie envers le chef Jack Layton? Volonté de réintégrer la dynamique politique canadienne ? Il y a eu un peu de tout cela dans le tsunami électoral qui a englouti des députés apparemment invulnérables et propulsé aux Communes de parfaits inconnus, dont une candidate unilingue anglophone habitant Ottawa, qui s'illusionnait si peu sur 
ses chances qu'elle s'était absentée durant la campagne pour effectuer un voyage touristique prépayé ! Dans un texte lourd de mépris qui mérite de passer à l'histoire, un idéologue souverainiste avait décrété que l'élection démontrait les limites de la démocratie, et traité pêle-mêle les Québécois ayant appuyé le NPD d'imbéciles, de " suiveux », de « moutons complexés » et pour finir de lemmings (" ces petits rongeurs nordiques qui sont à la fois stupides et solidaires au point de suivre leurs semblables lorsqu'ils se jettent en masse en bas d'une falaise ») (Lester N., 2011).

Gérés de main de maître par le lieutenant politique de Jack Layton, Tom Mulcair, les élus néo-démocrates ont pourtant honorablement rempli leur mandat. Devenue bien involontairement une célébrité à l'échelle québécoise, la nouvelle élue parachutée dont le succès avait suscité l'hilarité, Ruth Ellen Brosseau, apprit rapidement le français et conquit le cœur de ses nouveaux commettants de Berthier-Maskinongé au point d'être réélue triomphalement quatre ans plus tard malgré le reflux de la vague qui avait été à l'origine de sa promotion. Les défections qu'on avait prédites furent rares, et les problèmes d'ordre éthique encore moins ${ }^{2}$.

8 Après l'élection de 2011, les deux principaux partis d'opposition se choisirent des chefs issus du Québec. En mars 2012, l'ex-ministre libéral provincial et député d'Outremont, Tom Mulcair, fut élu chef du NPD, en remplacement de l'ontarien bilingue Jack Layton, décédé du cancer. Après le départ de Michael Ignatieff et l'intérim de Bob Rae, tous deux ontariens, Justin Trudeau, fils de l'ancien Premier ministre Pierre Trudeau, remporta en avril 2013 la course à la direction du parti libéral. Tout comme les autres Canadiens, les Québécois furent d'emblée sensibles à sa fraîcheur et à son charisme. En juin 2013, un sondage donnait à son parti $46 \%$ des intentions de vote au Québec, contre $26 \%$ au NPD, 15\% au Bloc Québécois et $8 \%$ aux Conservateurs ${ }^{3}$. Durant les six premiers mois de l'année 2015, cependant, l'étoile des Libéraux pâlit partout au pays, y compris au Québec, au profit du NPD, suite à quelques déclarations maladroites du chef libéral et à la victoire inattendue du NPD aux élections albertaines de mai 2015, qui semblait annoncer une nouvelle donne politique.

\section{Déroulement de la campagne}

9 Le postulat de départ de la campagne était celui d'une victoire néo-démocrate à l'échelle canadienne, doublée d'un triomphe de ce parti au Québec. L'engouement du pays pour les Libéraux semblait s'être évanoui au début de 2015. Partis troisièmes dans les sondages, les Libéraux ont effectué une lente et graduelle remontée à raison d'un point par semaine, ce qui les a menés au coude à coude avec les deux autres partis au milieu de la campagne ${ }^{4}$. Leur choix, risqué, de sacrifier l'équilibre budgétaire à la relance de l'économie, au rebours des deux autres partis (y compris du NPD, soucieux de rassurer l'électorat), leur a permis de se positionner comme le parti du changement.

C'est l'enjeu du niqab qui a permis de rompre l'égalité. En confirmant le 15 septembre l'illégalité d'une directive ministérielle interdisant à une nouvelle citoyenne de se voiler le visage durant la cérémonie de son assermentation, la division d'appel de la Cour Fédérale a provoqué une étincelle qui a ultimement fait dérailler la campagne du NPD au Québec. Menacé d'élimination, le Bloc Québécois a joué le tout pour le tout en diffusant une publicité ${ }^{5}$ ciblant cet enjeu, sur lequel le NPD était en porte-à-faux avec le sentiment prédominant au Québec et ailleurs au pays ${ }^{6}$. Adeptes du wedge politics, et apparemment sous l'influence d'un stratège australien expert en ces choses, les 
Conservateurs ont alimenté la controverse autant qu'ils ont pu, sentant qu'elle embarrassait leur principal adversaire là où il le fallait. L'appui des Québécois francophones au NPD a régulièrement reculé, et la vague orange de 2011 a fait place à un ressac orange, ce qui a permis aux Conservateurs de consolider leurs appuis, et au Bloc Québécois de refaire surface, stimulé par l'excellente performance de son chef lors des débats télévisés. Bien que favorables eux aussi au port du niqab, les Libéraux n'en ont guère pâti au Québec puisque le gros de leurs appuis y provenait des anglophones et des immigrants. La campagne canadienne en a été reconfigurée. Constatant que le NPD battait de l'aile dans sa forteresse québécoise et était donc de moins en moins susceptible de battre les Conservateurs, nombre de Canadiens favorables à un changement de gouvernement (environ $70 \%$ de l'électorat, selon tous les sondages) ont redirigé leurs appuis vers les Libéraux, qui ont pris la tête des intentions de vote.

\section{Les résultats}

11 Les Libéraux ont dépassé toutes les attentes au Québec avec $35,7 \%$ des voix et 40 sièges. Leur députation inclut suffisamment de députés qualifiés francophones pour bâtir une équipe ministérielle solide ${ }^{7}$. Personne ne leur dispute la palme du vainqueur parmi les électeurs francophones. Ils ont balayé l'île de Montréal, les Cantons de l'Est et l'Outaouais, tout en effectuant des percées inattendues ailleurs, décrochant deux sièges dans la ville de Québec et deux autres en Gaspésie.

Pour le NPD, la chute a été brutale : $25,4 \%$ des voix (contre $43 \%$ en 2011) et 16 députés (au lieu de 59). Les élus néo-démocrates sont éparpillés un peu partout sur le territoire. Nombre de vedettes sortantes ont cependant réussi à conserver leur siège : le chef Tom Mulcair dans Outremont, Alexandre Boulerice dans Rosemont, Hélène Laverdière dans Laurier Sainte-Marie, et Roméo Saganash dans l'Abitibi.

13 Les Conservateurs ont maintenu leurs appuis au Québec avec $16,7 \%$ des voix, et la débâcle du NPD leur a permis de porter le nombre de leurs sièges de 5 à 12, tous élus dans la ville de Québec et les régions rurales qui l'environnent. Ses ministres sortants ont tous été réélus.

14 Le Bloc Québécois a évité l'anéantissement complet qu'on lui prédisait en début de campagne en faisant élire 10 députés $^{8}$. C'est six de plus qu'en 2011, mais moins qu'il n'en fallait pour retrouver le statut de groupe parlementaire à la Chambre des communes, et ce relatif succès s'accompagne d'un recul de quatre points dans le suffrage populaire. Ses députés, tous novices à Ottawa, proviennent de la couronne montréalaise, de la région de Lanaudière et de la côte nord. Un seul a été élu sur l'île de Montréal ${ }^{9}$.

15 La participation électorale a été un peu moins élevée au Québec $(66,4 \%)$ que dans l'ensemble du pays $(68,5 \%)^{10}$, ce qui dans les deux cas représente cependant une progression significative en comparaison des chiffres de 2011 (62,9\% et $61,1 \%$ respectivement).

\section{Conclusion}

En défiant les attentes et en obtenant un gouvernement majoritaire, les Libéraux ont obtenu un bail de quatre ans à la tête du pays. En reconquérant le Québec, ils se sont 
assurés de pouvoir former un gouvernement plus représentatif des deux communautés linguistiques. En rupture avec l'atavisme traditionnel qui les amenait à appuyer massivement un parti depuis des lustres aux élections fédérales, les Québécois ont normalisé leur attitude et partagé leurs suffrages entre plusieurs partis, faisant la preuve qu'il existe chez eux un marché politique pour la droite dure des conservateurs et pour les indépendantistes les plus déterminés, aussi bien que pour les deux partis incarnant respectivement la social-démocratie et le libéralisme à l'américaine. Vingtcinq ans après l'échec de l'Accord du Lac Meech, 20 ans après la victoire du NON au référendum sur la souveraineté-partenariat, 10 ans après le scandale des commandites, 16 mois après la défaite inattendue du Parti Québécois en 2014, l'élection fédérale illustre le déclin de l'hégémonie nationaliste dans le débat public. Les espoirs des souverainistes se reporteront maintenant sur une percée du Parti Québécois de Pierre Karl Péladeau lors des élections provinciales prévues pour 2018. En attendant, le Canada a de bonnes chances de fêter son $150^{\mathrm{e} m e}$ anniversaire dans une relative sérénité.

\section{BIBLIOGRAPHIE}

Bourgault-Côté, Guillaume, « Trudeau premier de classe », Le Devoir, 21 juin 2013.

Brunet, Michel et Louis Massicotte, «CANADA - Histoire et politique », Encyclopedia Universalis, http://www.universalis.fr/encyclopedie/canada-histoire-et-politique/, page consultée le 23 octobre 2015.

« Députés qui ont traversé le parquet de la Chambre des communes ou qui ont changé d'affiliation politique », Parlinfo, http://www.parl.gc.ca/ParlInfo/lists/CrossedTheFloor.aspx? Language $=$ F\&Menu $=$ HOC-Politic $\&$ Name $=\&$ Section $=03 \mathrm{~d} 93 \mathrm{c} 58-$

f843-49b3-9653-84275c23f3fb\&Ministry=\&Parliament=1924d334-6bd0-4cb3-8793-cee640025ff6, page consultée le 22 octobre 2015.

Lessard, Denis et Martin Croteau, « Péladeau doute de la pertinence du Bloc », La Presse, 18 novembre 2014.

Lester, Normand, « Sains d'esprit, les Québécois ? », Le Devoir, 9 mai 2011.

Levitz, Stéphanie, « Les Québécois opposés au niqab dans les cérémonies de citoyenneté », Le Devoir, 24 septembre 2015.

« Liste de sondages sur l'élection fédérale canadienne de 2015 », Wikipedia, https://

fr.wikipedia.org/wiki/

Liste_de_sondages_sur_l\%27\%C3\%A9lection_f\%C3\%A9d\%C3\%A9rale_canadienne_de_2015, page consultée le 22 octobre 2015.

« Pétrole et niqab : une pub surprenante du Bloc (VIDÉO)», The Huffington Post, 18 septembre 2015, http://quebec.huffingtonpost.ca/2015/09/18/petrole-et-niqab--une-pub-surprenante-du-blocvideo_n_8161010.html, page consultée le 22 octobre 2015. 


\section{NOTES}

1. Le nombre de sièges du Québec est passé de 75 à 78, et le nombre total de députés à la Chambre des communes a augmenté de 308 à 338 (la plus forte augmentation depuis la Confédération), dans le cadre d'une redistribution opérée selon de nouvelles règles adoptées en 2011. En proportion, il s'agit pour le Québec d'un recul en termes relatifs, nécessité par la plus faible croissance de sa population.

2. Cinq députés québécois quittèrent le groupe parlementaire néo-démocrate pour s'égailler dans tous les horizons politiques. En comparaison, le Bloc québécois perdit trois de ses quatre députés durant la même période. Voir le site web PARLinfo.

3. Sondage Léger Marketing réalisé du 17 au 19 juin 2013 (Bourgault-Côté G., 2013).

4. Pour un graphique illustrant l'évolution des sondages, et pour accéder à chacun de ceux-ci, on se reportera à l'article de Wikipedia, «Liste de sondages sur l'élection fédérale canadienne de $2015 »$.

5. Pour visionner cette publicité, voir le site The Huffington Post: «Pétrole et niqab: une pub surprenante du Bloc (VIDÉO)». Un porte parole du NPD au Québec a ainsi commenté la publicité du Bloc : « Le Front National vient de faire son entrée dans la campagne ».

6. Les Conservateurs rendirent opportunément public un sondage gouvernemental confirmant le sentiment du public sur cet enjeu (Levitz S., 2015). L'opposition atteignait 93\% au Québec et $82 \%$ dans l'ensemble du pays.

7. Le nouveau Conseil des ministres, annoncé le 4 novembre 2015, compte sept Québécois parmi ses 31 membres. Trois proviennent de l'extérieur de Montréal.

8. Même le nouveau chef du Parti Québécois, Pierre Karl Péladeau, avait déclaré en novembre 2014 que le Bloc «ne servait strictement à rien, sinon à justifier le fédéralisme » (Lessard D.et Croteau M., 2014).

9. Le chef du parti, Gilles Duceppe, a été défait pour la deuxième fois dans sa propre circonscription et a annoncé peu après son retrait de la vie politique.

10. Chiffres officieux datés du 22 octobre 2015, susceptibles d'être légèrement rajustés à la hausse ultérieurement pour tenir compte des bulletins rejetés.

\section{AUTEUR}

\section{LOUIS MASSICOTTE}

Louis Massicotte est professeur titulaire au département de science politique de l'Université Laval, Québec, Canada. Il a enseigné auparavant à l'Université de Montréal et à l'American University de Washington DC. Spécialiste des institutions politiques, des parlements et des systèmes électoraux, il a été titulaire d'une chaire de recherche sur la démocratie et les institutions parlementaires. Parmi ses réalisations figurent Le Parlement du Québec depuis 1867 (2009), Establishing the Rules of the Game (2003 et Comment changer une constitution? (2011). Il a été associé au développement démocratique de treize pays, pour la plupart en Afrique francophone. Louis.massicotte@pol.ulaval.ca 